The Astronomical Journal, 122:591-597, 2001 August

(C) 2001. The American Astronomical Society. All rights reserved. Printed in U.S.A.

\title{
THE NEW TWO-IMAGE GRAVITATIONAL LENS SYSTEM CLASS B2319+051
}

\author{
D. Rusin AND D. R. MARLOW \\ Department of Physics and Astronomy, University of Pennsylvania, 209 South 33d Street, Philadelphia, PA 19104-6396 \\ M. Norbury, I. W. A. Browne, N. JaCkson, AND P. N. Wilkinson \\ Jodrell Bank Observatory, Lower Withington, University of Manchester, Macclesfield, Cheshire SK11 9DL, UK
}

C. D. FASSNACHT AND S. T. MYERS

National Radio Astronomy Observatory, P.O. Box O, Socorro, NM 87801

L. V. E. Koopmans, ${ }^{1}$ R. D. Blandford, T. J. Pearson, and A. C. S. Readhead

California Institute of Technology, Mail Stop 105-24, Pasadena, CA 91125

AND

\author{
A. G. DE BRUYN ${ }^{1}$ \\ Netherlands Foundation for Research in Astronomy, Postbus 2, NL-7990 AA Dwingeloo, Netherlands \\ Received 2000 October 1; accepted 2001 May 2
}

\begin{abstract}
We report the discovery of a new two-image gravitational lens system, CLASS B2319+051, from the Cosmic Lens All-Sky Survey. Radio imaging with the Very Large Array (VLA) and Multielement Radiolinked Interferometer Network (MERLIN) shows two compact components with a flux density ratio of $\simeq 5: 1$, separated by 1".36. Observations with the Very Long Baseline Array (VLBA) resolve each of the radio components into a pair of parity-reversed subcomponents. Hubble Space Telescope observations with the Near-Infrared Camera and Multi-Object Spectrometer (NICMOS) show a bright elliptical galaxy (G1) coincident with the radio position, and a second irregular galaxy (G2) 3".4 to the northwest. Previous spectroscopic studies have indicated that these galaxies are at different redshifts $\left(z_{\mathrm{G} 1}=0.624\right.$, $z_{\mathrm{G} 2}=0.588$ ). Infrared counterparts to the lensed radio components are not detected in the NICMOS image, and the source redshift has not yet been determined. Preliminary mass modeling based on the VLBA subcomponent data indicates that the lensing potential includes a strong external shear contribution. A VLA monitoring program is currently being undertaken to measure the differential time delay.

Key words: gravitational lensing
\end{abstract}

\section{INTRODUCTION}

The Cosmic Lens All-Sky Survey (CLASS; Myers et al. 1995 , 1999) seeks to discover new cases of gravitational lensing among flat-spectrum radio sources and ultimately produce the largest and best-studied sample of radioselected lenses. CLASS builds on the success of the JodrellVLA Astrometric Survey (JVAS; Patnaik et al. 1992; Browne et al. 1998; Wilkinson et al. 1998; King et al. 1999) and extends the search to weaker flux densities. The gravitational lens systems discovered by JVAS and CLASS are powerful tools for investigating a wide range of astrophysical and cosmological problems. First, arcsecond-scale lenses directly probe the inner several kiloparsecs of galaxies at intermediate redshift (Kochanek 1991) and can place vital constraints on their mass distributions. These studies indicate that the mass profiles of early-type lensing galaxies are close to isothermal (e.g., Kochanek et al. 1995; Cohn et al. 2001; Rusin \& Ma 2001). Second, measured time delays between the images of a lensed source, when combined with a well-constrained mass model, allow for a determination of the Hubble constant (Refsdal 1964). Thus far time delays have been measured for seven gravitational lens systems (Schechter et al. 1997; Kundić et al. 1997; Lovell et al. 1998; Wisotzki et al. 1998; Biggs et al. 1999; Fassnacht et al. 1999b; Koopmans et al. 2000) and favor a Hubble constant of $H_{0}=74 \pm 8 \mathrm{~km} \mathrm{~s}^{-1} \mathrm{Mpc}^{-1}$, assuming

\footnotetext{
${ }^{1}$ Also Kapteyn Astronomical Institute, Postbus 800, NL-9700 AV Groningen, Netherlands.
}

a flat cosmological model with $\Omega_{\Lambda}=0.7$ (Koopmans \& Fassnacht 1999). Third, the lensing rate in a systematic survey can place upper limits on the cosmological constant (Turner, Ostriker, \& Gott 1984; Turner 1990). Recent analyses favor $\Omega_{\Lambda} \leq 0.65$ for flat cosmologies (Kochanek 1996; Falco, Kochanek, \& Muñoz 1998; Quast \& Helbig 1999).

Sources in the first two phases of CLASS were selected from the 87GB $5 \mathrm{GHz}$ catalog (Gregory \& Condon 1991), with $S_{5} \geq 25 \mathrm{mJy}$ and spectral index $\alpha \geq-0.5$ (where $S_{v} \propto$ $v^{\alpha}$ ) between $5 \mathrm{GHz}$ and the $327 \mathrm{MHz}$ Westerbork Northern Sky Survey (WENSS; Rengelink et al. 1997) or the 365 $\mathrm{MHz}$ Texas Survey (Douglas et al. 1996). Recently, the sources in the CLASS sample were reselected using the 5 GHz GB6 catalog (Gregory et al. 1996) and the $1.4 \mathrm{GHz}$ NRAO VLA Sky Survey (NVSS; Condon et al. 1998), with a $5 \mathrm{GHz}$ cutoff of $30 \mathrm{mJy}$ and spectral index $\alpha \geq-0.5$ between 1.4 and $5 \mathrm{GHz}$. Spectral selection helps make CLASS a powerful and efficient lens survey. The flatspectrum CLASS sample is dominated by sources with intrinsically compact morphologies, which eases the identification of lenses and simplifies calculations of the statistical lensing rate. Furthermore, compact sources tend to be variable, thereby making possible the measurement of time delays from the light curves of lensed images.

The CLASS sample was observed using the Very Large Array (VLA) in A configuration at $8.4 \mathrm{GHz}$, which offers a resolution of $\simeq 250$ mas. Observations of $3 C 286$ were used to set the flux density scale. The VLA data were calibrated in the Astronomical Image Processing System (AIPS) and mapped using an automated script within the imaging 
package DIFMAP (Shepherd 1997). The data were then modeled with Gaussian components, which provides a quantitative description of the observed radio morphology. Sources modeled with multiple compact components are selected as preliminary lens candidates. These candidates are followed up with high-resolution radio observations using the Multielement Radio-linked Interferometer Network (MERLIN; resolution $\simeq 50$ mas at $5 \mathrm{GHz}$ ) and then the Very Long Baseline Array (VLBA; resolution $\simeq 5$ mas at $5 \mathrm{GHz}$ ) for the few surviving sources. The vast majority of the lens candidates are rejected on surface brightness and morphological grounds and are instead shown to be core-jet sources. Candidates survive the radio filter if their components have compact or correlated structure at the milliarcsecond scale. These are then followed up further with optical and/or near-infrared imaging and spectroscopy.

CLASS survey observations of over 13,000 sources were conducted in four phases (CLASS 1-4) from spring 1994 to summer 1999 and are now complete. The first phase of CLASS observations (CLASS 1) has yielded five new lens systems: B0128 +437 (Phillips et al. 2000), B0712+472 (Jackson et al. 1998), B1600+434 (Jackson et al. 1995), B1608 + 656 (Myers et al. 1995), and B1933 + 503 (Sykes et al. 1998). The second series of observations (CLASS 2) has produced another five lenses: B0739+366 (Marlow et al. 2001), B1127+385 (Koopmans et al. 1999), B1555+375 (Marlow et al. 1999b), B2045+ 265 (Fassnacht et al. 1999a), and the one presented in this paper, B2319+051. Two additional lens systems have recently been discovered during the third phase of CLASS observations (CLASS 3): B1152+199 and B1359+154 (Myers et al. 1999; Rusin et al. 2000). Radio follow-up observations of the remaining CLASS 3 and CLASS 4 candidates are nearly complete.

Here we report the discovery of a new two-image gravitational lens from CLASS 2: B2319+051. In $\S 2$ we describe radio observations of the system with the VLA, MERLIN and VLBA. Section 3 presents near-infrared imaging with the Hubble Space Telescope (HST). Preliminary mass modeling of B2319+051 is described in $\S 4$. Section 5 summarizes our results and discusses future work.

\section{RADIO OBSERVATIONS}

B2319+051 was observed on 1995 August 13 during the second phase of the CLASS survey observations. The 8.4 $\mathrm{GHz}$ discovery snapshot map has an rms noise of $330 \mu \mathrm{Jy}$ beam $^{-1}$ and is displayed in Figure 1. The source consists of two compact components with flux densities of $27.4 \pm 0.3$ mJy (component A) and $5.0 \pm 0.3 \mathrm{mJy}$ (component $\mathrm{B}$ ) in a north-south orientation, separated by 1".36. Follow-up VLA $1.4,5,8.4$, and $15 \mathrm{GHz} A$ configuration observations were performed on 1999 July 29 to investigate the spectral properties of the radio components. The flux density scale was set by observations of the calibrator source $\mathbf{J} 2355+498$. The VLA data sets were calibrated in AIPS using the standard procedure and analyzed in DIFMAP. In each case the visibility data were fitted to a pair of compact Gaussian components using several iterations of modelfitting and phase-only self-calibration (solution interval of 0.5 minutes). The component flux densities are given in Table 1, and the radio spectra are plotted in Figure 2. The radio spectra exhibit striking similarity, with overall spectral indices between 1.4 and $15 \mathrm{GHz}$ of $\alpha_{1.4}^{15}=-0.66 \pm 0.01$

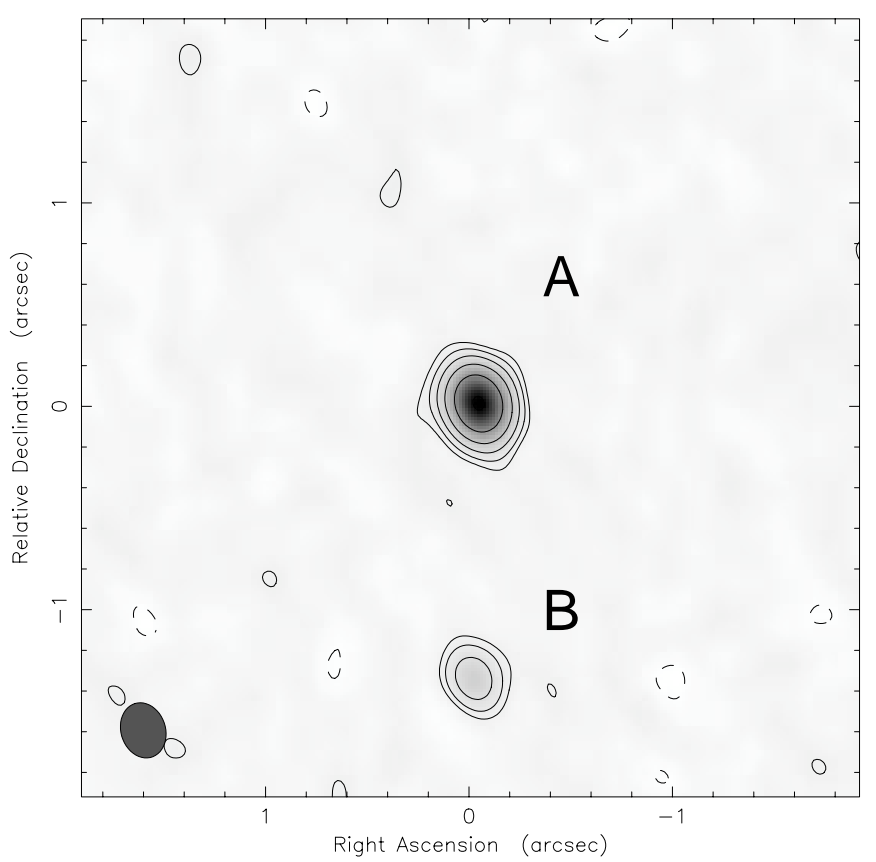

FIG. 1.-VLA $8.4 \mathrm{GHz}$ discovery snapshot observation of B2319+051 taken 1995 August 29 . The lowest contour is at $\pm 3 \%$ of the map peak of $26.3 \mathrm{mJy}$ beam $^{-1}$, and contour levels increase by factors of 2 . The synthesized beam is $0.276 \times 0$ ". 219 at 17.4 . The data have been naturally weighted.

(A) and $\alpha_{1.4}^{15}=-0.61 \pm 0.05(\mathrm{~B})$, respectively. This argues against the identification of $\mathrm{A}$ and $\mathrm{B}$ as either two independent quasars or components of a core-jet structure. However, nearly identical radio spectra would be expected for images of a gravitationally lensed source.

MERLIN $5 \mathrm{GHz}$ observations of B2319+051 were performed on 1996 December 26 for a total integration time of $1 \mathrm{hr}$ and again on 1998 February 24 for $9 \mathrm{hr}$ to improve the

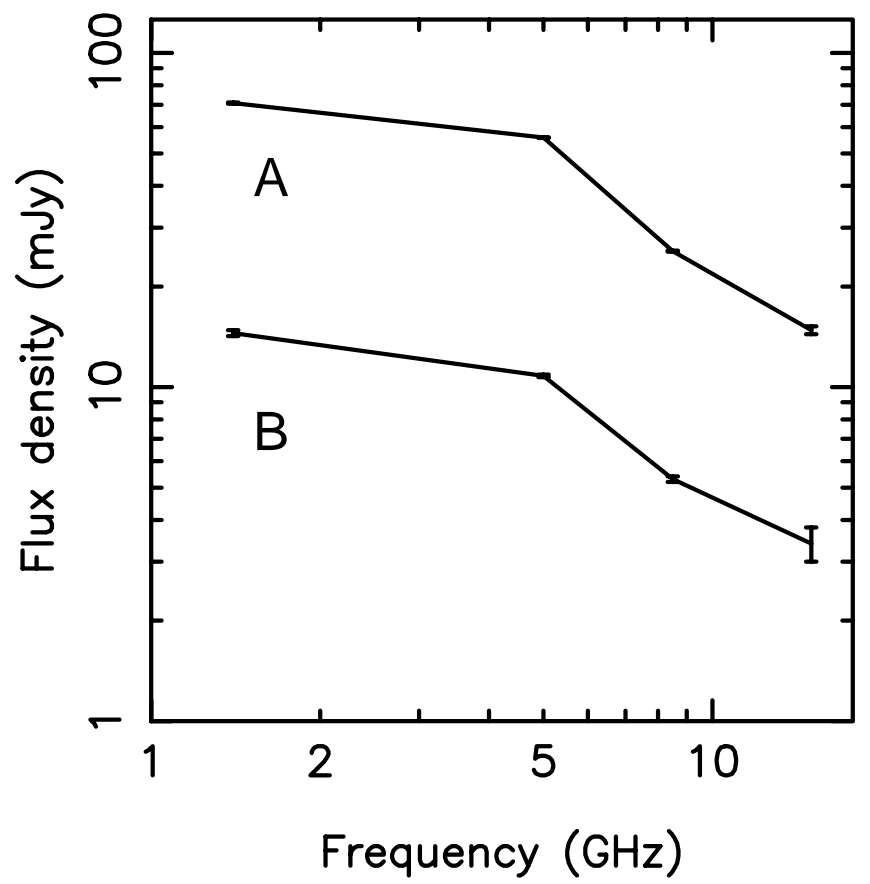

FIG. 2.-Component radio spectra based on the VLA 1.4, 5, 8.4, and 15 GHz data of 1999 July 29. 
TABLE 1

DATA FOR THE 1999 JULY 29 OBSERVATION

\begin{tabular}{lccrr}
\hline \hline Component & $S_{1.4}$ & $S_{5}$ & \multicolumn{1}{c}{$S_{8.4}$} & \multicolumn{1}{c}{$S_{15}$} \\
\hline A $\ldots \ldots \ldots \ldots$ & $70.8 \pm 0.3$ & $55.8 \pm 0.1$ & $25.5 \pm 0.1$ & $14.8 \pm 0.4$ \\
B $\ldots \ldots \ldots \ldots$ & $14.5 \pm 0.3$ & $10.8 \pm 0.1$ & $5.3 \pm 0.1$ & $3.4 \pm 0.4$ \\
\hline
\end{tabular}

NotE.-VLA Component Flux Densities in millijanksys at 1.4, 5, 8.4, and $15 \mathrm{GHz}$. Errors in the flux densities are taken to be equal to the rms noise of the respective maps.

surface brightness sensitivity. Observations of 3C 286 were used to set the flux density scale. The data were calibrated in AIPS and imaged in DIFMAP by repeating a cycle of cleaning and phase-only self-calibration, starting with long solution intervals (40 minutes) and gradually decreasing to a minimum interval of 2 minutes. Once the model had sufficiently converged, an amplitude self-calibration was performed using a solution interval of 30 minutes. The final map has an rms noise of $70 \mu \mathrm{Jy}$ beam $^{-1}$ and is shown in Figure 3. The data were modeled by two compact Gaussian components with flux densities of $44.0 \pm 0.1 \mathrm{mJy}(\mathrm{A})$ and $8.8 \pm 0.1 \mathrm{mJy}(\mathrm{B})$. No further emission was detected down to the $3 \sigma$ level of the maps. The compactness of each radio component in the MERLIN map offers further evidence for the lensing hypothesis.

VLBA $5 \mathrm{GHz}$ observations of $\mathrm{B} 2319+051$ were performed on 1997 August 3. The observations were obtained using five separate snapshots, each of seven minutes duration, over a range of hour angles to synthesize $u v$ coverage. The system was reobserved at $5 \mathrm{GHz}$ on 2000 September 18 for $11 \mathrm{hr}$ to improve the sensitivity. In each case, phase referencing was implemented using the nearby calibrator source $\mathrm{J} 2320+052$. Fringe fitting was performed on $\mathrm{J} 2320+052$ and the solutions were transferred directly to $\mathrm{B} 2319+051$. The calibrated data were then imaged in DIFMAP, using several iterations of cleaning and phaseonly self-calibration, with a solution interval of 5 minutes.

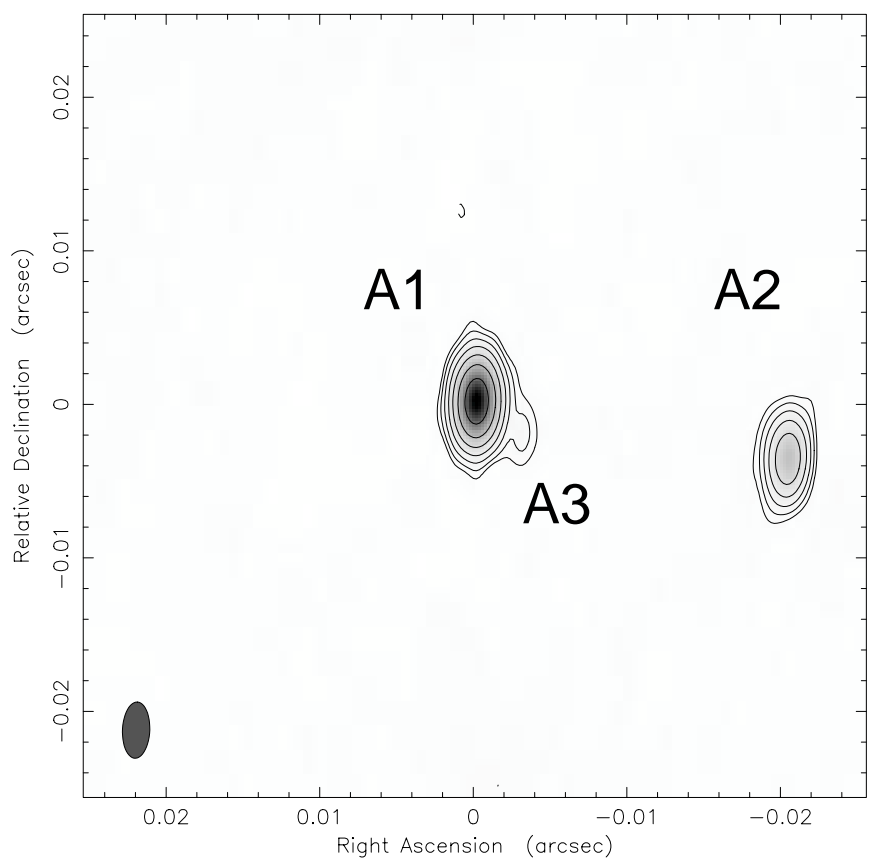

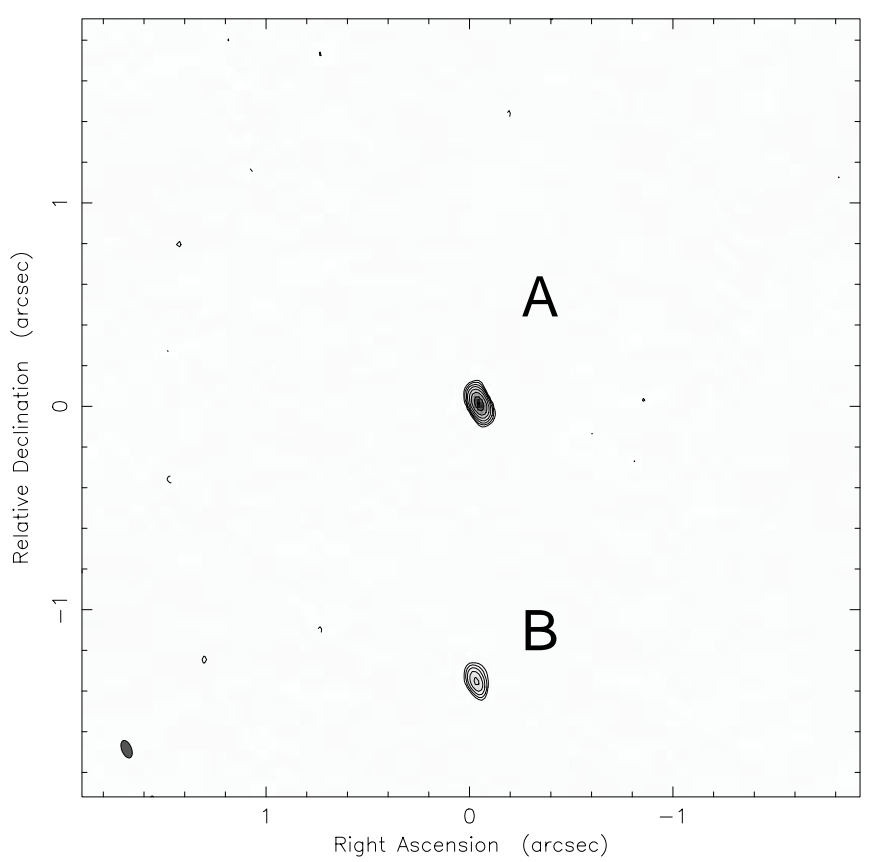

FIG. 3.-MERLIN $5 \mathrm{GHz}$ observation of B2319+051 taken 1998 February 24 . The lowest contour is at $\pm 1 \%$ of the map peak of $42.4 \mathrm{mJy}$ beam $^{-1}$, and contour levels increase by factors of 2 . The synthesized beam is $0.091 \times 00^{\prime \prime} .048$ at $21^{\circ} .9$. The data have been naturally weighted.

Maps from the deep observation are presented in Figure 4 and have an rms noise of $50 \mu \mathrm{Jy}_{\text {beam }}^{-1}$. Each of the radio components is resolved into a pair of compact subcomponents (A1 and A2, B1 and B2), indicative of a "coreknot" source morphology. In addition, we identify a very weak emission feature (A3) as the beginning of a radio jet connecting $\mathrm{A} 1$ and $\mathrm{A} 2$. A corresponding feature is not obvious in $\mathrm{B}$, but its detection is greatly hindered by the decreased flux density and angular size of the component.

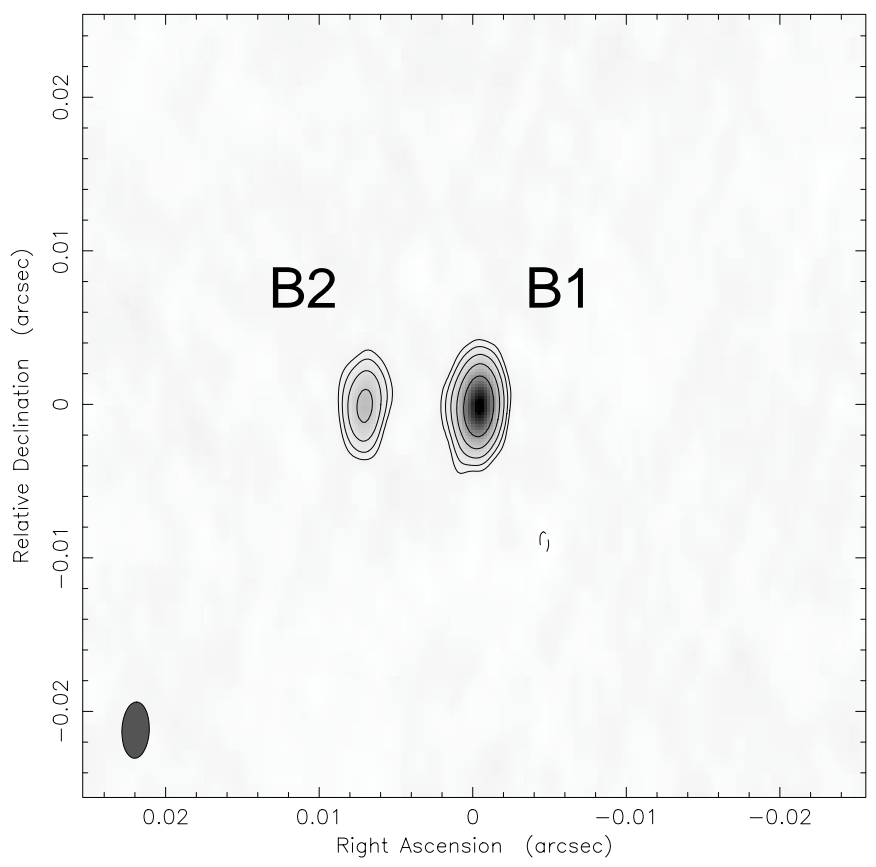

FIG. 4.-VLBA $5 \mathrm{GHz}$ observation of B2319+051 taken 2000 September 18 . The beam is $3.7 \times 1.8$ mas at -2.5 . The data have been naturally weighted. Left: component A. The lowest contour is at $\pm 1 \%$ of the map peak of $30.1 \mathrm{mJy}^{-1}$ beam ${ }^{-1}$, and contour levels increase by factors of 2 . (b) Right: component B. The lowest contour is at $\pm 3 \%$ of the map peak of $5.9 \mathrm{mJy} \mathrm{beam}^{-1}$, and contour levels increase by factors of 2 . 
TABLE 2

VLBA DATA FOR B2319+051

\begin{tabular}{lcccc}
\hline \hline Component & $\begin{array}{c}\Delta \text { R.A. } \\
(\operatorname{arcsec})\end{array}$ & $\begin{array}{c}\Delta \text { decl. } \\
(\operatorname{arcsec})\end{array}$ & $\begin{array}{c}S_{5} \\
(\mathrm{mJy})\end{array}$ & $\begin{array}{c}S_{1.7} \\
(\mathrm{mJy})\end{array}$ \\
\hline A1 $\ldots \ldots \ldots$. & 0 & 0 & 31.0 & 42.7 \\
$\mathrm{~A} 2 \ldots \ldots \ldots$. & -00203 & -00037 & 8.3 & 14.4 \\
$\mathrm{~A} 3 \ldots \ldots \ldots$. & -00030 & -00024 & 0.8 & 9.4 \\
$\mathrm{~B} 1 \ldots \ldots \ldots$. & +00113 & -13638 & 6.2 & 9.2 \\
B2 $\ldots \ldots \ldots \ldots$ & +00188 & -13638 & 1.9 & 2.8 \\
\hline
\end{tabular}

Notes.-Positions are from the 2000 September $185 \mathrm{GHz}$ observation and are offset from R.A. $23^{\mathrm{h}} 21^{\mathrm{m}} 40^{\mathrm{s}} 8015$, decl. $+05^{\circ} 27^{\prime} 37^{\prime \prime} .2252(\mathrm{~J} 2000.0)$. Flux densities are listed for both the 5 and $1.7 \mathrm{GHz}$ VLBA observations. Model-fitting errors are approximately \pm 0.1 mas for positions $\mathrm{A} 1, \mathrm{~A} 2, \mathrm{~B} 1$, and $\mathrm{B} 2$. The positional error for $A 3$ is substantially larger ( $\simeq 1$ mas). Uncertainties on the flux densities are $\simeq 5 \%$.

B1 does, however, appear to be slightly extended along the east-west direction, possibly signaling the presence of this emission feature. While the maps we present employ natural visibility weighting, the higher resolution obtained by switching to uniform weighting is more than offset by the decreased sensitivity and did not improve the analysis. The visibility data were well fitted by a total of five compact $(<1$ mas) Gaussian components, using iterated cycles of modelfitting and phase-only self-calibration. The positions and flux densities of the subcomponents are listed in Table 2.

Deep $1.7 \mathrm{GHz}$ VLBA observations of B2319+051 were obtained on 1999 August 12. The total integration time was $12 \mathrm{hr}$. Phase referencing and fringe fitting were performed with the nearby JVAS source J $2322+082$. The calibration and imaging procedures were identical to those described above. The resulting maps have an rms noise of $55 \mu \mathrm{Jy}$ beam $^{-1}$ and are displayed in Figure 5. The substructure observed in the $1.7 \mathrm{GHz}$ maps matches that detected at 5 $\mathrm{GHz}$. The data were modeled by five Gaussian components that closely correspond to those derived from the $5 \mathrm{GHz}$

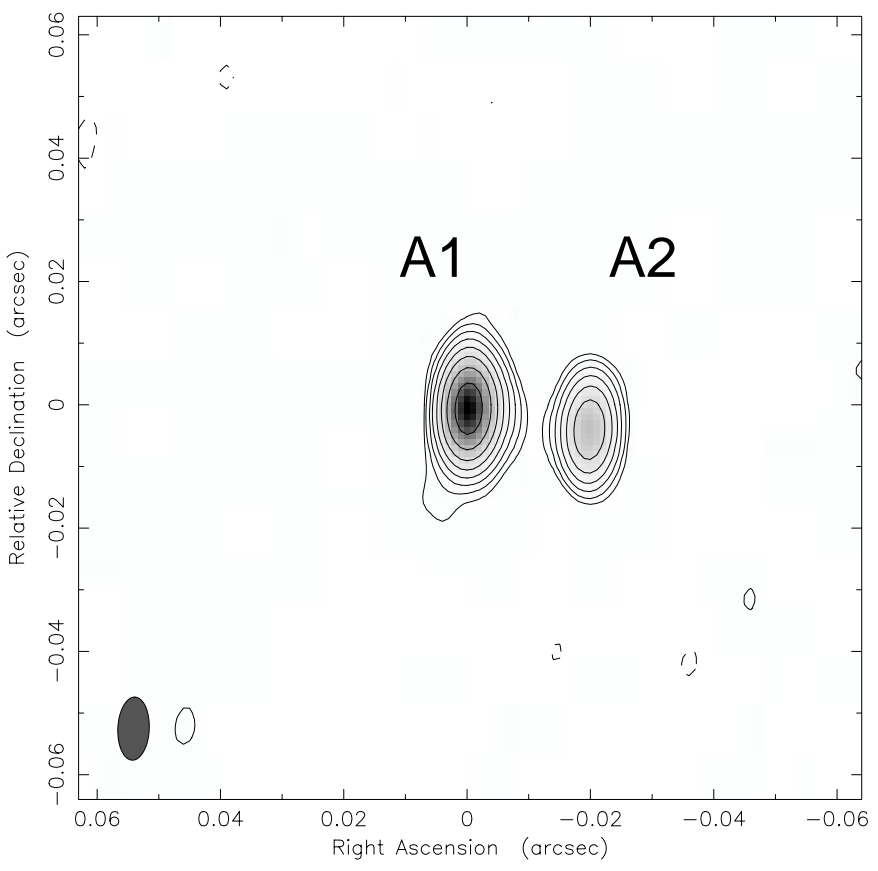

observations. Table 2 lists the flux densities of these components. The residual map was searched for evidence of faint compact emission features that could be associated with a third "odd" image of the source (Rusin \& Ma 2001), but none were found down to the detection threshold.

The milliarcsecond-scale substructure of the B2319+051 radio components contains the unmistakable signature of gravitational lensing. First, components $\mathrm{A}$ and $\mathrm{B}$ share nearly identical morphologies, as would be expected for images of a common background source. Second, the relative inversion of the radio components is a textbook example of lensing-induced parity reversal (e.g., Schneider, Ehlers, \& Falco 1992). Third, the brighter component (A) covers a larger angular size, consistent with the conservation of surface brightness by gravitational lensing. Taken together, these observations offer compelling evidence that $\mathrm{B} 2319+051$ is a gravitational lens system.

Finally, we searched for changes in the substructure of the $\mathrm{B} 2319+051$ radio images by comparing the relative positions of the core and knot subcomponents in the 1997 and 2000 VLBA $5 \mathrm{GHz}$ data. The likelihood of detecting evolving superluminal jets is expected to be enhanced for lensed radio sources, as magnification would increase the apparent transverse velocity. Jet evolution may offer useful constraints on the mass model, in particular on the local magnification matrices, but this effect has not yet been observed in any gravitational lens system. We find that the A1-A2 and B1-B2 separations in the 1997 and 2000 data sets are each consistent to within 0.1 mas. Our tests indicate that this is close to the reliability limit of DIFMAP model fitting. Consequently, there is no evidence for evolving radio substructure in B2319+ 051 at this time.

\section{HST IMAGING AND ASTROMETRY}

$H S T$ observations of B2319+051 were obtained on 1998 May 30 with the Near-Infrared Camera and Multi-Object

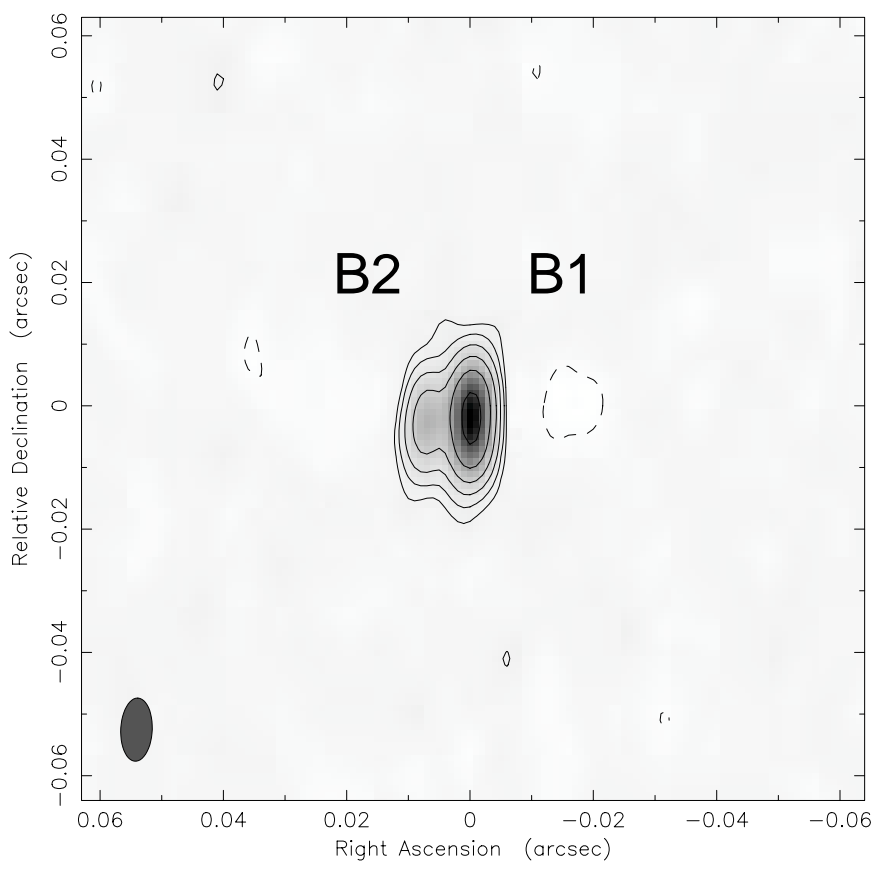

Fig. 5.-VLBA $1.7 \mathrm{GHz}$ observation of B2319+051 taken 1999 August 12 . The beam is $10.3 \times 5.1$ mas at $-2{ }^{\circ} 6$. The data have been naturally weighted. Left: component A. The lowest contour is at $\pm 0.5 \%$ of the map peak of $46.8 \mathrm{mJy}_{\text {beam }}^{-1}$, and contour levels increase by factors of 2 . Right: component B. The lowest contour is at $\pm 2.5 \%$ of the map peak of $6.3 \mathrm{mJy}^{-1}$ beam $^{-1}$, and contour levels increase by factors of 2 . 
TABLE 3

\begin{tabular}{|c|c|c|c|}
\hline Component & $\begin{array}{c}\Delta \mathrm{R} . \mathrm{A} . \\
(\operatorname{arcsec})\end{array}$ & $\begin{array}{c}\Delta \text { decl. } \\
(\operatorname{arcsec})\end{array}$ & $m_{\mathrm{F} 160 \mathrm{~W}}$ \\
\hline G1 ......... & 0 & 0 & 18.2 \\
\hline G2a ........ & -2257 & 2421 & 191 \\
\hline G2b ........ & -2957 & 1777 & 19.1 \\
\hline
\end{tabular}

Note.-The errors on the positions are \pm 0 " 015 . The errors on the magnitudes are \pm 0.15 .

Spectrometer (NICMOS). The F160W filter, which is centered at $1.6 \mu \mathrm{m}$ and corresponds roughly to ground-based $H$ band, was used. The observations used the NIC2 camera, with a detector scale of 75 mas pixel $^{-1}$ and a field of view of $19{ }^{\prime \prime} 2 \times 19^{\prime \prime} .2$. The total exposure time was $2624 \mathrm{~s}$. The data were subjected to the standard NICMOS calibration pipeline, involving bias and dark current subtraction, flat-field correction, cosmic-ray removal, and photometric calibration. The final image is displayed in Figure $6 a$. A bright elliptical galaxy (G1) is observed at the position of the radio system, but no counterparts to the radio images are detected. This suggests a very optically faint lensed source, similar to B1127+385 (Koopmans et al. 1999) or B1933 + 503 (Marlow et al. 1999a). The observation of a galaxy close to the expected position does, however, satisfy an important criterion of the lensing hypothesis. A second, irregular galaxy (G2) lies 3".4 to the northwest of G1. A contour plot of the NICMOS image (Fig. $6 b$ ) clearly shows that $\mathrm{G} 2$ is composed of two subcomponents ( $\mathrm{G} 2 \mathrm{a}$ and $\mathrm{G} 2 \mathrm{~b}$ ).

Photometry and relative astrometry were performed on G1 and G2 (Table 3). The elliptical surface brightness profile of $\mathrm{G} 1$ has an axis ratio of $0.63 \pm 0.02$ (at the outmost isophote) and a position angle of $52^{\circ} \pm 5^{\circ}$. The integrated F160W magnitudes are 18.2 and 19.1 for G1 and G2, respectively, with an uncertainty of \pm 0.15 . Recent spectro-

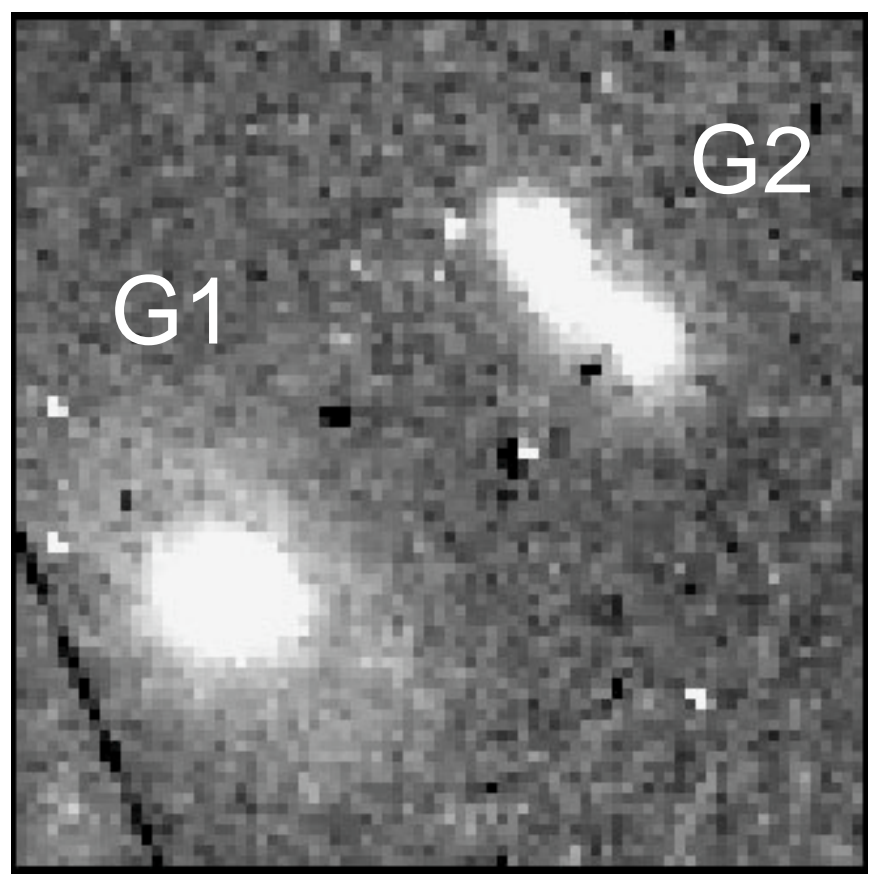

scopic observations using the Low Resolution Imaging Spectrograph (LRIS) on the W. M. Keck telescope have demonstrated that the galaxies are not physically associated: $z_{\mathrm{G} 1}=0.624$ and $z_{\mathrm{G} 2}=0.588$ (Lubin et al. 2000). The spectrum of G1 is consistent with an early-type galaxy. G1 has approximately the luminosity of an E/S0 $L^{*}$ galaxy, including a $K$-correction but no evolutionary correction (Poggianti 1997). The spectrum of G2 shows strong Balmer absorption lines which, when combined with the irregular morphology, suggest an interacting or merging system.

The absence of detectable counterparts to the lensed radio components means that we cannot determine the positions of the galaxies relative to the images by using any one data set. We therefore attempted to extract the absolute coordinates of G1 from the Keck observations of Lubin et al. (2000). Reference stars were selected from the USNO A2.0 Catalog (Monet et al. 1996) and the astrometric solutions were calculated using Judy Cohen's COORDINATES program, which takes into account the distortions introduced by the LRIS optics. Eight unsaturated reference stars were selected for the program, and the estimated final rms position error is 0.45 . Unfortunately, the saturation of many reference stars in the Keck image makes subpixel accuracy impossible with the current data set. With the solutions from the COORDINATES program, we have determined the position of G1 to be R.A. $=23^{\mathrm{h}} 21^{\mathrm{m}} 40^{\mathrm{s}} .817$, decl. $=+05^{\circ} 27^{\prime} 36^{\prime \prime} .57$ (J2000.0). Based on the VLBA positions of the lensed radio components, this would place G1 at $(0.27,-0$.'66) relative to A1, roughly between the two lensed images.

\section{MASS MODELING}

B2319+051 presents a greater modeling challenge than many two-image gravitational lens systems because of the lack of detected optical or infrared counterparts to the

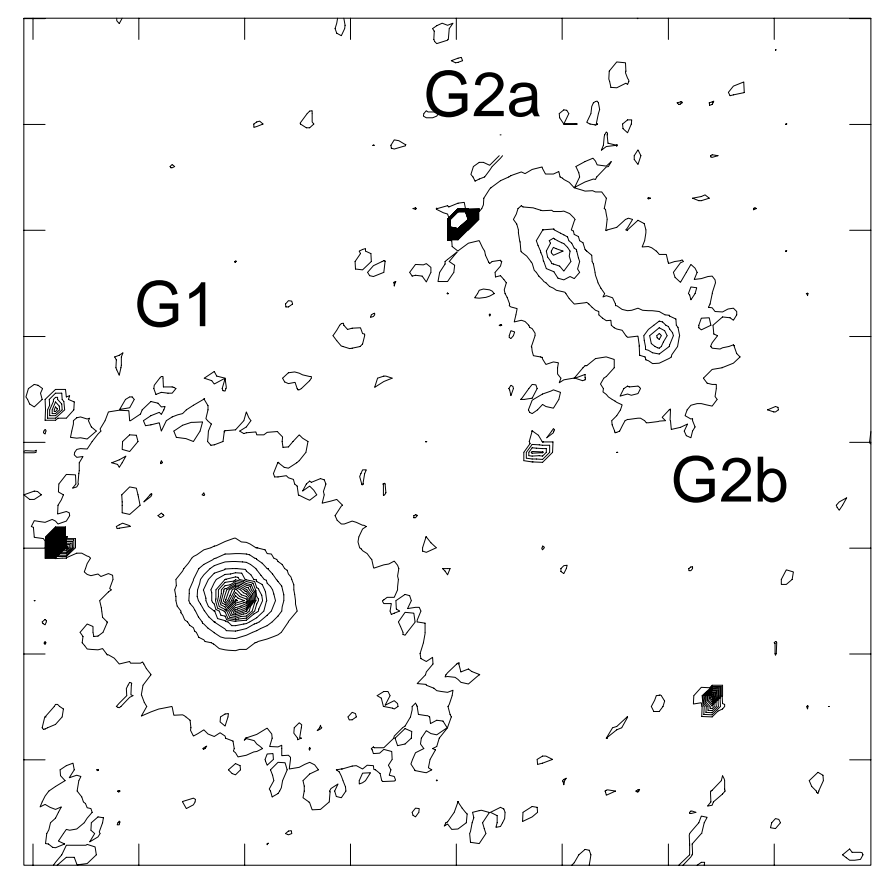

FIG. 6.-NICMOS F160W images of B2319+051. North is up; east is left. The area shown is $6.0 \times 6.0$. Left, final map; right, contour map. The contour levels are $(1,2,3, \ldots, 28)$ times rms noise in the image. Note the absence of any counterparts to the lensed radio images, and the two surface brightness peaks of $\mathrm{G} 2$. 
lensed radio components. Though the astrometry is not adequate to fix robustly the position of the lensing galaxy at this time, the milliarcsecond-scale radio substructure offers a sufficient number of constraints to test simple mass models. In this analysis we use the eight coordinates $(x, y)$ of $\mathrm{A} 1, \mathrm{~A} 2, \mathrm{~B} 1$, and B2, as well as the two $5 \mathrm{GHz}$ flux density ratios $r_{1}=\left|S_{\mathrm{B} 1} / S_{\mathrm{A} 1}\right|$ and $r_{2}=\left|S_{\mathrm{B} 2} / S_{\mathrm{A} 2}\right|$. Modeling was performed with an image plane minimization (Kochanek 1991), which optimized the fit statistic

$$
\begin{aligned}
\chi^{2}= & \sum_{i=A 1, A 2, B 1, B 2}\left[\frac{\left(x_{i}^{\prime}-x_{i}\right)^{2}}{\Delta x_{i}^{2}}+\frac{\left(y_{i}^{\prime}-y_{i}\right)^{2}}{\Delta y_{i}^{2}}\right] \\
& +\sum_{i=1,2} \frac{\left(r_{i}^{\prime}-r_{i}\right)^{2}}{\Delta r_{i}^{2}},
\end{aligned}
$$

where primed quantities are model-predicted and unprimed quantities are observed. We assumed a tolerance of $\Delta x=\Delta y=0.1$ mas on the image positions and $5 \%$ on each of the flux densities $(\Delta r \simeq 7 \%)$ to account for possible modeling errors and source variability. A flat $\Omega_{\Lambda}=0.7$ universe with $H_{0}=100 h \mathrm{~km} \mathrm{~s}^{-1} \mathrm{Mpc}^{-1}$ and a source redshift of $z=1.5$ were assumed for all calculations.

We first modeled B2319+ 051 by using a singular isothermal ellipsoid mass distribution (SIE; Kormann, Schneider, \& Bartelmann 1994) with surface density

$$
\Sigma(x, y)=\frac{\sigma^{2}}{2 G} \frac{\sqrt{f}}{\sqrt{x^{2}+f^{2} y^{2}}},
$$

where $\sigma$ is the line-of-sight velocity dispersion and $f$ is the projected axial ratio. Including the four parameters required to describe the unlensed source subcomponents, the model has nine free parameters. Consequently, the number of degrees of freedom (NDF) is one. Not only does the model offer a rather poor fit to the radio data $\left(\chi^{2}\right)$ $\mathrm{NDF}=3.1$ ), but the mass distribution is predicted to lie at a position angle of $\simeq-30^{\circ}$, nearly orthogonal to that of the observed surface brightness of G1. This is unlikely to be a consistent scenario, as modeling studies have demonstrated that lensing mass distributions are typically well aligned with the light (Keeton, Kochanek, \& Falco 1998). Fixing the mass distribution at the observed galaxy position angle of $52^{\circ}$ leads to an unacceptable fit. Even when the coordinate uncertainties are relaxed to a very liberal 1 mas, $\chi^{2} /$ $\mathrm{NDF}=14$ for $\mathrm{NDF}=2$, with mismatch of the subcomponent positions dominating the fit statistic. We interpret the poor performance of the single-galaxy model as evidence that external shear is significantly influencing the lensing potential. Because the SIE model requires an ellipticity oriented at $\simeq-30^{\circ}$, galaxies along this axis are likely to be responsible for the shear. Lubin et al. (2000) detect several galaxies, including G2, to the northwest of the system. These are likely candidates for the perturbing mass.

We therefore expanded the lens model to include an external shear field of constant direction and magnitude. To reduce the number of free parameters and ensure a constrained model, we set the position angle and axial ratio of the SIE to the observed values of the G1 surface brightness. The SIE + shear model provides a much improved fit to the radio data $\left(\chi^{2} / \mathrm{NDF}=0.45\right.$ with $\left.\mathrm{NDF}=1\right)$. The optimized model parameters are listed in Table 4 . The critical structure of the model is plotted in Figure 7. The preferred shear field has magnitude $\gamma=0.14$ and position angle $-22^{\circ}$. G2 is unlikely to account for all the model-predicted shear, unless
TABLE 4

\begin{tabular}{|c|c|}
\hline Parameter & Model \\
\hline$\ldots \ldots \ldots \ldots$ & 0.63 \\
\hline$\sigma_{\mathrm{G} 1} \ldots \ldots \ldots \ldots$ & $237.7 \mathrm{~km} \mathrm{~s}^{-1}$ \\
\hline$(x, y)_{\mathrm{G} 1} \ldots \ldots \ldots$ & $\left(+0^{\prime \prime} 2085,-0^{\prime \prime} 7891\right)$ \\
\hline P.A. ${ }_{\mathrm{G} 1} \ldots \ldots \ldots \ldots$ & $+52^{\circ}$ \\
\hline$\gamma \ldots \ldots \ldots \ldots \ldots$ & 0.141 \\
\hline P.A. ${ }_{\gamma} \ldots \ldots \ldots \ldots$ & -22.4 \\
\hline$(x, y)_{\mathrm{src} 1} \ldots \ldots \ldots$ & $\left(+0^{\prime \prime} 2809,-0^{\prime \prime} 7606\right)$ \\
\hline$(x, y)_{\mathrm{src} 2} \ldots \ldots \ldots$ & $\left(+0^{\prime \prime} .2787,-0^{\prime \prime} .7583\right)$ \\
\hline$\mu_{\mathrm{A} 1, \mathrm{~A} 2} \ldots \ldots \ldots \ldots$ & $+12.73,+11.82$ \\
\hline$\mu_{\mathrm{B} 1, \mathrm{~B} 2} \ldots \ldots \ldots \ldots$ & $-2.62,-2.61$ \\
\hline$\Delta t \ldots \ldots \ldots \ldots \ldots$ & $17.8 h^{-1}$ days \\
\hline$\chi^{2} / \mathrm{NDF} \ldots \ldots \ldots$ & 0.45 \\
\hline
\end{tabular}

Best-Fit SIE + Shear Model Parameters FOR B2319+051

NoTES.-All values assume a flat $\Omega_{\Lambda}=0.7$ cosmology. Listed are the fixed surface density axial ratio, $f_{\mathrm{G} 1}$, velocity dispersion, $\sigma_{\mathrm{G} 1}$, coordinates, $(x, y)_{\mathrm{G} 1}$, and fixed position angle, P.A. ${ }_{G 1}$, for the SIE; magnitude, $\gamma$, and position angle, P.A., , for the external shear; and recovered source coordinates, $(x, y)_{\text {src }}$, predicted magnifications, $\mu$, and time delay, $\Delta t$. The image positions are reproduced almost exactly and are not listed.

it has a much higher mass-to-light ratio than G1. (An isothermal mass distribution at 3".4 requires a velocity dispersion of $\simeq 260 \mathrm{~km} \mathrm{~s}^{-1}$ to produce a shear field with $\gamma=0.14$.) The position of the lensing galaxy $\left(0,21,-00^{\prime \prime} 79\right)$ agrees well with that derived from our LRIS astrometry. The predicted time delay between components $\mathrm{A}$ and $\mathrm{B}$ is $\simeq 18 h^{-1}$ days for a flat $\Omega_{\Lambda}=0.7$ cosmology.

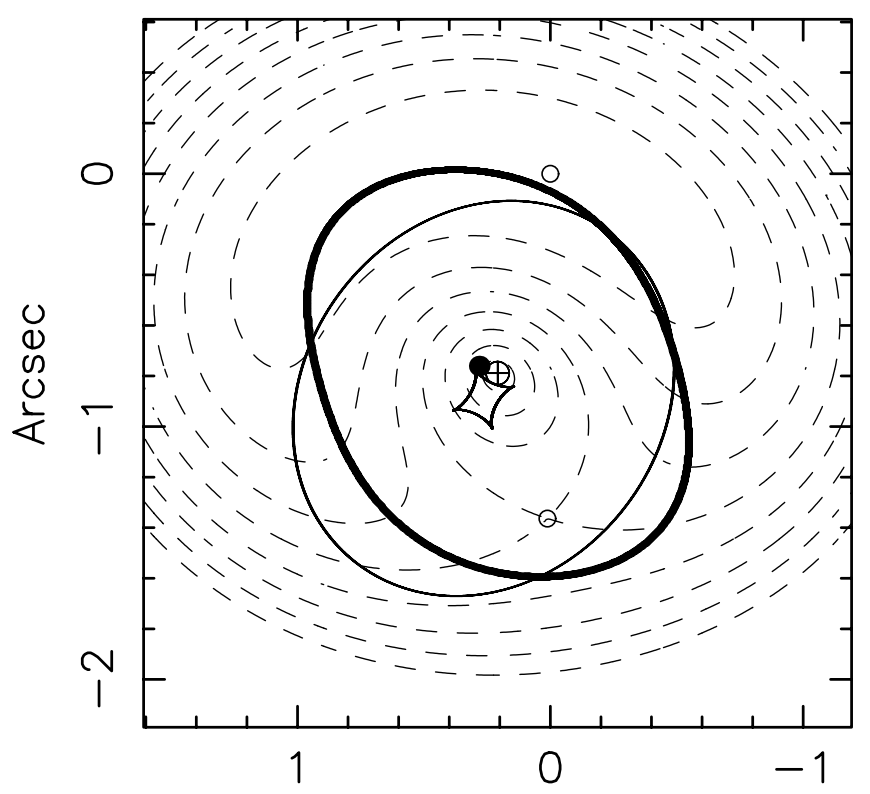

Arcsec

FIG. 7.-Critical curve (thick line) and caustics (thin lines) of the SIE + shear lens model, showing the recovered source position (filled circle), the positions of the images (open circles), and the center of the SIE (circle with cross-hairs). Dashed lines denote contours of constant time delay in increments of $4.45 h^{-1}$ days outward from the global minimum at image A. The caustics are offset from the position of the lensing galaxy because of our choice of "centering" the shear at $(0,0)$. This has no effect on the optimized model parameters or predicted time delay. 


\section{SUMMARY AND FUTURE WORK}

VLA and MERLIN observations of CLASS B2319+051 show two compact radio components separated by 1".36. Both components have virtually identical radio spectra between 1.4 and $15 \mathrm{GHz}$, consistent with their being images of a single background source. VLBA $5 \mathrm{GHz}$ observations resolve each of the components into a pair of compact subcomponents. VLBA $1.7 \mathrm{GHz}$ observations confirm this finding. The similar morphologies, consistent angular sizes, and relative inversion of the radio components provide powerful evidence to support the lensing hypothesis. HST/ NICMOS imaging has revealed a bright elliptical galaxy at the expected position, along with a second irregular galaxy 3.4 to the northwest. Infrared counterparts to the radio components were not detected in this observation. However, the radio spectra and substructure, along with the identification of a lensing galaxy, argue that B2319+051 is, indeed, a genuine gravitational lens system.

Preliminary mass modeling has demonstrated that an isolated galaxy is unable to account for the VLBA substructure while remaining consistent with the properties of the lensing galaxy G1. Because an isolated SIE is required to be oriented nearly perpendicular to the observed surface brightness of $\mathrm{G} 1$, nearby galaxies may be contributing significant ellipticity to the lensing potential. The introduction of external shear allows for an excellent fit to the data, even when the position angle and axial ratio of the SIE are fixed to match the G1 surface brightness. The favored shear direction is consistent with mass distributions located to the northwest of G1, as detected by Lubin et al. (2000). Considering our inability to fix robustly the position of the primary lensing mass relative to the lensed images, it is quite remarkable that the simple substructure in the $\mathrm{B} 2319+051$ radio components not only strongly excludes an isolated galaxy model but also selects a reasonable direction for the external shear field.

Of paramount importance to future studies of $\mathrm{B} 2319+051$ is the detection of optical or infrared counterparts to the lensed images. The positions of these images relative to the lensing galaxy will provide an essential check for our preliminary modeling attempt, while offering additional constraints for the construction of more realistic mass models. The detection of these images would also be a first step toward measuring the source redshift, which is a vital ingredient for Hubble constant determination. $\mathrm{B} 2319+051$ is currently being monitored as part of a large VLA program to measure time delays in JVAS and CLASS gravitational lens systems.

We thank the staffs of the VLA, MERLIN, and VLBA for their assistance during our observing runs. The National Radio Astronomy Observatory is a facility of the National Science Foundation operated under cooperative agreement by Associated Universities, Inc. MERLIN is operated as a National Facility by the University of Manchester, on behalf of the UK Particle Physics and Astronomy Research Council. This research used observations with the Hubble Space Telescope, obtained at the Space Telescope Science Institute, which is operated by Associated Universities for Research in Astronomy, Inc., under NASA contract NAS 5-26555. D. R. acknowledges funding from the Zaccheus Daniel Foundation. This work was supported in part by European Commission TMR Programme, Research Network Contract ERBFMRXCT 96-0034 CERES.

\section{REFERENCES}

Biggs, A. D., Browne, I. W. A., Helbig, P., Koopmans, L. V. E., Wilkinson, P. N., \& Perley, R. A. 1999, MNRAS, 304, 349

Browne, I. W. A., Wilkinson, P. N., Patnaik, A. R., \& Wrobel, J. M. 1998, MNRAS, 293, 257

Cohn, J. D., Kochanek, C. S., McLeod, B. A., \& Keeton, C. R. 2001, ApJ, in press

Condon, J. J., Cotton, W. D., Greisen, E. W., Yin, Q. F., Perley, R. A., Taylor, G. B., \& Broderick, J. J. 1998, AJ, 115, 1693

Douglas, J. N., Bash, F. N., Bozyan, F. A., Torrence, G. W., \& Wolfe, C. 1996, AJ, 111, 1945

Falco, E. E., Kochanek, C. S., \& Muñoz, J. A. 1998, ApJ, 494, 47

Fassnacht, C. D., et al. 1999a, AJ, 117, 658

Fassnacht, C. D., Pearson, T. J., Readhead, A. C. S., Browne, I. W. A., Koopmans, L. V. E., Myers, S. T., \& Wilkinson, P. N. 1999b, ApJ, 527, 498

Gregory, P. C., \& Condon, J. J. 1991, ApJS, 75, 1011

Gregory, P. C., Scott, W. K., Douglas, K., \& Condon, J. J. 1996, ApJS, 103, 427

Jackson, N., et al. 1995, MNRAS, 274, L25

. 1998, MNRAS, 296, 483

Keeton, C. R., Kochanek, C. S., \& Falco, E. E. 1998, ApJ, 509, 561

King, L. J., Browne, I. W. A., Marlow, D. R., Patnaik, A. R., \& Wilkinson, P. N. 1999, MNRAS, 307, 225

Kochanek, C. S. 1991, ApJ, 373, 354

. 1995, ApJ, 445, 559

- 1996, ApJ, 466, 638

Koopmans, L. V. E., et al. 1999, MNRAS, 303, 727

Koopmans, L. V. E., de Bruyn, A. G., Xanthopoulos, E., \& Fassnacht, C. D. 2000, A\&A, 356, 391

Koopmans, L. V. E., \& Fassnacht, C. D. 1999, ApJ, 527, 513

Kormann, R., Schneider, P., \& Bartelmann, M. 1994, A\&A, 284, 285

Kundić, T., et al. 1997, ApJ, 482, 75

Lovell, J. E. J., Jauncey, D. L., Reynolds, J. E., Wieringa, M. H., King, E. A., Tzioumis, A. K., McCullough, P. M., \& Edwards, P. G. 1998, ApJ, 508, L51
Lubin, L. M., Fassnacht, C. D., Readhead, A. C. S., Blandford, R. D., \& Kundić, T. 2000, AJ, 119, 451

Marlow, D. R., Browne, I. W. A., Jackson, N., \& Wilkinson, P. N. 1999a, MNRAS, 305,15

Marlow, D. R., et al. 1999b, AJ, 118, 654

2001, AJ, 121, 619

Monet, D., et al. 1996, USNO-SA2.0 (Washington: US Naval Obs.)

Myers, S. T., et al. 1995, ApJ, 447, L5

1999, AJ, 117, 2565

Patnaik, A. R., Browne, I. W. A., Wilkinson, P. N., \& Wrobel, J. M. 1992, MNRAS, 254, 655

Phillips, P. M., et al. 2000, MNRAS, 319, L7

Poggianti, B. M. 1997, A\&AS, 122, 399

Quast, R., \& Helbig, P. 1999, A\&A, 344, 721

Refsdal, S. 1964, MNRAS, 128, 307

Rengelink, R. B., Tang, Y., de Bruyn, A. G., Miley, G. K., Bremer, M. N., Roettgering, H. J. A., \& Bremer, M. A. R. 1997, A\&AS, 124, 259

Rusin, D., Hall, P. B., Nichol, R. C., Marlow, D. R., Richards, A. M. S., \& Myers, S. T. 2000, ApJ, 533, L89

Rusin, D., \& Ma, C.-P. 2001, ApJ, 549, L33

Schechter, P. L., et al. 1997, ApJ, 475, L85

Schneider, P., Ehlers, J., \& Falco, E. E. 1992, Gravitational Lenses (Berlin: Springer)

Shepherd, M. C. 1997, in ASP Conf. Ser. 125, Astronomical Data Analysis Software and Systems IV, ed. G. Hunt \& H. E. Payne (San Francisco: ASP), 77

Sykes, C. M., et al. 1998, MNRAS, 301, 310

Turner, E. L. 1990, ApJ, 365, L43

Turner, E. L., Ostriker, J. P., \& Gott, J. R. 1984, ApJ, 284, 1

Wilkinson, P. N., Browne, I. W. A., Patnaik, A. R., Wrobel, J. M., \& Sorathia, B. 1998, MNRAS, 300, 790

Wisotzki, L., Wucknitz, O., Lopez, S., \& Sorensen, A. N. 1998, A\&A, 339, L73 